Historic, Archive Document

Do not assume content reflects current scientific knowledge, policies, or practices. 



\section{RETAIL LIST}

Spring, 1926

\section{GLADIOLUS}

BULBS

Choicest Varieties from

World's Leading Originators

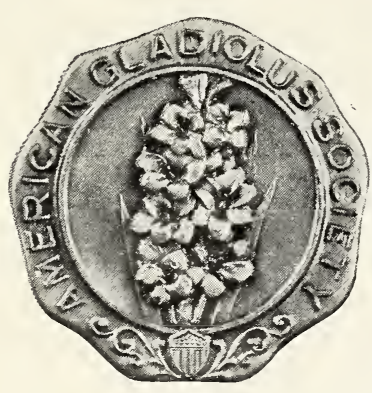

American Gladiolus Society Silver Medal

New York, August, 1924

\section{B. HALSEY SPENCER}

79 HOPKINS PLACE

LONGMEADOW, MASSACHUSETTS 
Announcing Introduction

\section{7 ORCHID LADY}

Large orchid lavender blooms with silvery sheen. Fall plant and spike. Early. A magnificent variety. MEDAL WINNER 


\section{PRICE LIST}

ADORATION, Large ruffled saffron.....

Large Half-size Bulblets

Each Each Doz. 100

*ADRIATIC, Wine blue, red throat............ AH, Apricot pink and vellow.

ALBANIA, Very fine white.

$\$ .50 \$ .25 \$ .50 \$ 3.00$

AL'TON, Ruffled orange.

* ANNA EBEPIUS, Purple and fine

*A. B. KUNDERD, Ruffled cream and pink

*ARIZONA ROSE, Large rose pink.......

*AVALON, Ruffled blush white.

ANTHONY ZONKER, Ruffled salmon pink, blotch

BARON HULOT, Pansy purple

*B. L. SMITH, Lavender pink, cream throat

BEVIER, Salmon rose, cream throat.....

BLACK JOE, Clear deep red, fine............

BLUE LILY, Light violet blue.

CARDINAL'PRINCE, Clear cardinal red

CAPTAIN BOYNTON, Lavender on white ground

CARMEN SYLVA, One of the best whites

$.15 \quad-15 \quad .75$
.25

$\begin{array}{llll}.25 & .15 & .35 & 2.00\end{array}$

$\begin{array}{llll}.20 & .10 & .25 & 1.00\end{array}$

$\begin{array}{llll}.25 & .15 & .25 & 1.50\end{array}$

$\begin{array}{llll}.15 & .08 & .15 & .75\end{array}$

$\begin{array}{llll}.35 & .20 & .35 & 2.00\end{array}$

$\begin{array}{lllr}.10 & .05 & .10 & .50\end{array}$

$\begin{array}{llll}.15 & .08 & .15 & .60\end{array}$

$\begin{array}{llll}.40 & .20 & - & - \\ .10 & .05 & -\end{array}$

$\begin{array}{llll}.15 & .08 & .15 & .50\end{array}$

$\begin{array}{llll}.15 & .08 & .15 & .50\end{array}$

$\begin{array}{llll}.25 & .15 & - & -\end{array}$.

$3.00 \quad 1.50 \div-$

$\begin{array}{llll}.60 & .30 & .75 & 3.00\end{array}$

$\begin{array}{llll}.15 & .08 & .15 & .60\end{array}$

$\begin{array}{llll}.15 & .08 & .15 & .60\end{array}$ fine

CATHERINE COLEMAN, Coral pink..

CIRCE, Early mahogany red, good...........

CLASSIC, Shell pink and white, red blotch

*CRIMSON GLOW, Large crimson, one of the best...

CRINKLES, Ruffled peach blossom shade

*CLAREMONT, Light yellow deep yellow throat

CAPT, MILES STANDISH, Pure self red

* IIANA, A real fine red.

*D's AMERICAN BEAUTY

DOROTHY McKIBBON, Ruffled rose pink

DORRITT, Large lavender pink, showy

DREAM, Ruffled salmon red.

DR. F. E. BENNETT, Large vivid orange scarlet

DR. VAN FLEET, Tall rose with yellow throat

DR. ELKINS, Large white, grape purple throat

DR. J. H. NEELEY, Tall white tinted pink

*DR. R. T. JACKSON, Dark velvety red

*E, J. SHA YLOR, Fine ruffled deep rose

E. G. HILL, Flesh pink, yellow throat

ELIZABETH TABOR, Rose pink crimsoa bloteh

*ELORA, Large white, red lines in throat

ELKHART, Vjolet purnle deeper throat

*EVELYN KIPTLAND, Exceptional rose pink

EXCELLO, A Pendelton seedling...............

EXQUISI'E, Self color American Beauty Rose

EUROPA, Nearest pure white

$\begin{array}{llll}1.00 & .75 & 1.00 & -\end{array}$

$\begin{array}{llll}.25 & .15 & .25 & 1.00\end{array}$

$.20 \quad .10 \quad .20$

$\begin{array}{llll}.10 & .05 & .10 & .50\end{array}$ 
EARLY SUNRISE, Very large sunrise red

* FAIR COLUMBIAN, Showy lilac, tall FERN KYLE, Ruffled creamy white.....

FLORA, Beautiful all yellow.

*FREDA, Large geranium pink, new.........

F. J. SYMMES, Ruffled dark salmon......

GERTRUDE ERREY, New pink from Australia

GERALDINE FARRAR, Pale lavender violet

GIAN'T MYRTLE, Rare pale rose pink

GIANT NYMPH, Very large light rose pink

GLENDALE, Like the best red cyclamen

GOLD, Considered best yellow...

GOLD ELSIE Pure bright yellow

GOLDEN GLORY, Ruffled dark throat

GOLDEN MEASURE, Larger, taller than Gold

GOLD THROAT, Blood red and gold.....

*HENRY C. GOEHL, Large white crimson blotch

*HERADA, Pure mauve, rare beauty.....

HENRY FORD, Rhodamine purple, large

HERMES, Ruffled cherry pink....

HOLLYHOCK, White, red blotch, round like flower.

HELGA, Tall early salmon rose light throat

INDIAN MAID, (True variety) Peach pink

*INDIAN SUMMER, Large late light lavender

ILLUMINATOR, Deep rose red....-..-..-........

IMMENSITY, Large bright salmon........

J. A. CARBONE, Orange salmon, yellow throat

JACOBA VON BEIJEREN, Light purple, self color.

JACK LONDON, Salmon, orange stripes

JENNY LIND, Clear salmon, cream throat

JOE COLEMAN, Large ruffled red, different

J. T. PIRIE, Mahogany brown, odd........

JOERGS WHITE, Very large, prize winner

KASSON, Lavender rose pink ruffled......

LAVENDER AMERICA, Beautiful flower

$\begin{array}{rrrr}.20 & .10 & -\bar{r} & -\overline{90} \\ .20 & .10 & .20 & .90 \\ .50 & .25 & .50 & 2.50 \\ .10 & - & .10 & .50 \\ .25 & .15 & .25 & - \\ .10 & - & - & -\end{array}$

5.00

$2.50 \quad 1.50 \quad-\quad-$

$\begin{array}{rrrr}.75 & .50 & 1.00 & 8.00\end{array}$

$\begin{array}{llll}.35 & .20 & 1.25 & 8.00\end{array}$

$\begin{array}{llll}.35 & .20 & \overline{35}\end{array}$

$\begin{array}{llll}.25 & .15 & .35 & 2.00 \\ .50 & - & - & \end{array}$

$\begin{array}{llll}.15 & \overline{10} & \overline{25} & 1 . \overline{50}\end{array}$

$\begin{array}{llll}.15 & .10 & .25 & 1.50\end{array}$

$.25 \quad .15-1.50$

$\begin{array}{llll}.35 & .20 & -\overline{10} & \overline{60}\end{array}$

$\begin{array}{llll}1.00 & .75 & 1.00 & 6.00\end{array}$

$\begin{array}{rrrr}.50 & .25 & .50 & -\end{array}$

$1.00-\quad-$

$\begin{array}{llll}.15 & .10 & .15 & .60\end{array}$

$.50-2-$

$\begin{array}{llll}.50 & .25 & .50 & 3.00\end{array}$

$\begin{array}{lllr}.75 & - & .90 & - \\ .25 & .15 & .25 & 1.50\end{array}$

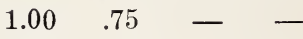

$.25 \quad .15 \quad-\quad$

$.10-\quad-$

$\begin{array}{llll}.30 & .15 & .30 & 2.00\end{array}$

$\begin{array}{llll}.15 & .10 & .15 & .90\end{array}$

$.75 \quad .50 \quad-\quad-$

$\overline{.25} \quad \overline{15} \quad \overline{.25} \quad$ -

$.35--$

$\begin{array}{llll}.50 & \overline{15} & \overline{.25} & 1 . \overline{50}\end{array}$ let blotch, fine.

LEOT A, Giant rose pink, white throat

* LE MARECHAL FOCH, Large enchantress pink

LILAC GLORY, Soft lilac rose pink......

LONGFELLOW, La France pink, large, grand

LOS ANGELES, Delicate pink, cerise feather

LOUISE, Bright lavender, wine throat, good

*LUSTRE. Dark orange vermillion, SUPREME RED

$.06 \quad .03 \quad-\quad .35$

$.50 \quad .25 \quad-\quad$

$.50-\quad-$

$.50-.50-$

$.15-\quad-$

$\begin{array}{llll}.20 & .10 & .20 & 1.25\end{array}$ 
MME. MOUNET SULLY, Cream white, bright red blotch...

MADISON COOPER, Fine dark red, real good

MARIE KUNDERD, Large ruffled snow white .....................................................

MARIET'TA, Large salmon tinged orange

* IARSHAL FOCH, Ruffled salmon pink, grand

* IIARY FENNELL, Deep lilac, yellow throat

* IIARY PICKFORD, Creamy white, soft yellow throat.................................................

MISS MADISON, Delicate light pink, new

MISS SPOKANE, Very good dark salmon

MONA LISA, Soft rose pink.

IR. W. H. PHIPPS, Wonder glad, flesh pink

* MRS. DR. NORTON, Cream and pink, none better ...............................................

MRS. F. C. PETERS, Lilac rose, deep blotch

*MRS. F. PENDLETON, Shell pink,

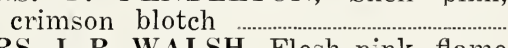

MRS. J. R. WALSH, Flesh pink, flame red blotch

MRS. H. E. BOTHIN, Ruffled flesh salmon, blotch

MRS. G. W. MOULTON, Giant violet red

MRS. KR. PRESTGARD, New pure white

MRS. LAURA SHEDD SCHWEPPE, Peach blossom pink.

MRS. LEON DOUGLAS, Begonia rose, stripped scarlet

MRS. WM. E. CLARK, Salmon pink, blotch

MURIEL, Best of the so called blues......

*MYSTIC, Flaked rose pink, white throat, fine

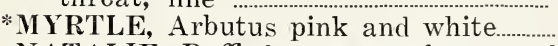

NATALIE, Ruffled orange salmon, red blotch

NORMA TALMADGE, Large light yellow, new

OBELISQUE, Large cream, yellow lip, fine

OPALESCENT, Pale lavender.

ORANGE FLAME, Ruffled vermillion scarlet

ORANGE GLOW, Tall orange salmon

PARAMOUNT, Choice pink and cream

PEACH ROSE, Clear rose pink, a beauty

*PINK LILY, Ruffled deep rose pink, fine

* PINK WONDER, Large light rose pink

PURPLE GLORY, Deepest velvety maroon

*PRIDE OF LANCASTER, Orange salmon, fine

POLLYA Y …....................................................

"PYTHIA, Large red that is different, winner..

Each Each Doz. 100

\begin{tabular}{|c|c|c|c|}
\hline 30 & - & - & - \\
\hline .35 & .20 & .35 & 1.50 \\
\hline .50 & .25 & .50 & 2.75 \\
\hline .00 & .50 & - & \\
\hline .20 & .10 & - & \\
\hline .10 & .05 & .10 & 50 \\
\hline .15 & .10 & .15 & 0 \\
\hline .50 & - & .50 & \\
\hline $\begin{array}{l}.10 \\
.15\end{array}$ & .05 & .10 & $\begin{array}{l}.50 \\
.75\end{array}$ \\
\hline .50 & 1.50 & 2.50 & \\
\hline .10 & .05 & - & .50 \\
\hline .35 & - & .35 & 2.00 \\
\hline .06 & .03 & - & .40 \\
\hline .25 & .15 & .25 & 1.50 \\
\hline .10 & .05 & - & .50 \\
\hline .15 & .10 & .15 & .75 \\
\hline .50 & - & - & \\
\hline 1.50 & 1.00 & 1.50 & 10.00 \\
\hline .60 & .40 & .60 & \\
\hline $\begin{array}{l}.25 \\
.25\end{array}$ & - & $\overline{.25}$ & 1.50 \\
\hline .15 & .10 & $.15^{\prime}$ & .50 \\
\hline .25 & - & - & \\
\hline 1.50 & - & - & - \\
\hline $\begin{array}{l}.50 \\
.30\end{array}$ & .30 & - & - \\
\hline 1.00 & .75 & 1.50 & 10.00 \\
\hline $\begin{array}{r}.35 \\
1.00\end{array}$ & - & - & \\
\hline .40 & - & .40 & \\
\hline .15 & .10 & .15 & \\
\hline .15 & .10 & .15 & \\
\hline .35 & .20 & .35 & 1.50 \\
\hline .20 & .10 & .20 & 1. \\
\hline & 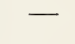 & - & \\
\hline .80 & .15 & .30 & \\
\hline
\end{tabular}


RED FIRE, Magnificent fiery red........... REV. EWBANK, Light porcelain blue RICHARD DIENER, Rose salmon, yellow center

RED COPPER, Deep salmon rose, flaked blue

*REMEMBRANCE, Salmon rose, deeper throat, fine

*RARITY, Choice lavender rose...................

*ROSE ASH, Ashes of Roses, tall, large

*ROSE GLORY, Pure rose pink, ruffled

ROSE, Red blotched rose

RUTH HUNTINGTON, Pale lilac, very fine

R. J. KUNDERD, Large orange vermillion

ROSELLA, Near old rose

*SCARLANO, Dazzling scarlet orange

*SCARLET PRINCEPS, Fine scarlet.....

SCARLET WONDER, Very large clear red, great

SHEILA, Early tall light salmon...............

SHEILA'S SISTER, Larger than Sheila

SHELL PINK, A very fine variety........

SEABROOK GEM, Light rose lavender

SIDNEY PLUMMER, Soft yellow, amber lip

SUPERBA, Deep rose overlaid scarlet

SHARON ROSE, Beautiful rose, scarce

*SWEET LAVENDER, Early light lavender, blotch

SWEET ROSE, Soft deep rose pink, fine

SULPHUR GLOW, Ruffled yellow blotch, fine

SPLENDORA, Deep wine black, well liked

SUNNYMEDE, Rare shade orange yellow

THISTLE, Fine ruffled rose red, beauty

TWILIGHT, Creamy buff yellow and pink

TYREAN BEAUTY, American Beauty shade

UHLAN CHIEF, Brilliant scarlet, fine

VIOLET BEAUTY, Cerise violet, deeper throat

VIOLET GLORY, Deep violet, self color

VIRGINIA HALE, Creamy salmon rose

WHITE GLORY, White, iris blue throat

WHITE WONDER, Large fine white clear

*WILBRINK, Early large flesh pink ........

WOLVERINE, Flesh, crimson throat

WM. KENT, Creamy shell pink, yellow center

WM. BADGER, Large salmon rose, cream throat

YELLOW GLORY, Fine yellow, purple throat

*YOUELLS FAVORITE, Rosy lavender pink, fine $\begin{array}{llll}.50 & .25 & .50 & 2.50\end{array}$

$\begin{array}{llll}.15 & .10 & .15 & .60\end{array}$

$\begin{array}{llll}.50 & .25 & .50 & 4.00\end{array}$

$\begin{array}{llll}.25 & .15 & .25 & 1.25\end{array}$

$\begin{array}{llll}.15 & .10 & .15 & .75\end{array}$

$\begin{array}{llll}.25 & .15 & .25 & 1.25\end{array}$

$\begin{array}{llll}.20 & .10 & .20 & 1.00\end{array}$

$\begin{array}{llll}.15 & .10 & .15 & .75\end{array}$

$.25-.25 \quad-$

$\begin{array}{llll}.30 \quad .20 \quad- & -\end{array}$

$.35--$

$.25-2-$

$.10 \quad .05-.50$

$\begin{array}{llll}.15 & .10 & .15 & .50\end{array}$

$\begin{array}{llll}.50 & .25 & .50 & 2.00\end{array}$

$.15-\quad-.75$

$.15-\quad-.75$

$.25 \quad .15-7-$

$.25-\quad-1.00$

$\begin{array}{llll}.35 & .20 & .35 & 2.00\end{array}$

$1.00-$ -

$1.00--$

$\begin{array}{llll}.25 & .15 & .25 & 1.50\end{array}$

$4.00--$

$\begin{array}{llll}.25 & .15 & .25 & 1.25\end{array}$

$\begin{array}{llll}.15 & .10 & .15 & .75\end{array}$

$.50-2-$

$.25 \quad .15-$

$\begin{array}{llll}.35 & .20 & .35 & 1.75\end{array}$

$.25-\quad-\quad-$

$\begin{array}{llll}.25 & .15 & .25 & 1.50\end{array}$

$.20-\quad-\quad-$

$\begin{array}{llll}.15 & .10 & .15 & .75\end{array}$

$.75--$

$\begin{array}{llll}.15 & .10 & .15 & .75\end{array}$

$\begin{array}{llll}.15 & .10 & .15 & .75\end{array}$

$.10-\quad-.50$

$.10-\quad-.35$

$2.00--\quad-$

$3.50--$

$\begin{array}{llll}.25 & .15 & .25 & -\end{array}$

$\begin{array}{llll}.15 & .10 & .15 & .75\end{array}$ 


\section{PRIMULINUS VARIETIES}

ALICE TIPLADY, Choice orange sal-

Large Half-size Bulblets

Large
Each
Each

ALTAIR, Tall salmon saffron

*ARLON, Large orange saffron

*ARGO, Large pink and cream.

BUT'TERBOY, Tall fine yellow.

*CAPELLA, A great fine red, very tall

EDA, Very large orange.

$\begin{array}{rrrr}.10 & - & - & .50 \\ .10 & - & - & .50 \\ .10 & - & - & .50 \\ .10 & - & - & .50 \\ .25 & .15 & .25 & 1.00 \\ .15 & .10 & .15 & .75 \\ .15 & .10 & .15 & .75 \\ 1.00 & .50 & 1.00 & 5.00\end{array}$

ETH. WILLAMSON, new light purple fine

FROSTY (Spencer), Light rose red, flaked gray

GOLDEN GLEAM, Light orange, very good

GLADNESS, Unusual lavender prim.....

JEWELL, Light salmon, yellow throat

JUNO, Orange

LILAC OLD ROSE, Very attractive......

MAIDEN'S BLUSH, Blush pink

MING TOY, Beautiful buff and yellow

*MYRA, Giant deep salmon, yellow throat

MIDSUMMER DREAM, Large geranium red

*NIGHTINGALE, Softest rose pink

ORANGE QUEEN, Fine orange yellow

ROSE MIST, Old rose, edged gray........

SAGINAW, Very large buff..

SALMON BEAUTY, Deep salmon, yellow throat

SALMON BUFF, Throat of soft yellow

SONIA, Bright red, good.

SOUVENIR, Deep yellow, very fine

*SVEA, Very dainty light pink, yellow throat

SUNRISE, Yellowish orange, pretty......

*THOTH, Fine white prim, yellow throat

*TOPAZ, Salmon pink and buff

TWINKLES, Soft salmon rose..

ULRICA, Fine yellow and orange-.......

WHITE BUTTERFLY, Fine white prim

WHITE PRIM (Spencer), White and cream

$\begin{array}{rrrr}.35 & - & - & - \\ 1.00 & .50 & - & - \\ .25 & .15 & .25 & 1.25 \\ .15 & .10 & .15 & .75 \\ .15 & .10 & - & .75 \\ .25 & .15 & - & - \\ .35 & - & - & - \\ .15 & .10 & .15 & .75 \\ .25 & .15 & .25 & 1.25 \\ .10 & .05 & .10 & .50 \\ .15 & .10 & .15 & .75 \\ .15 & .10 & .15 & .75 \\ .35 & .20 & .35 & - \\ 2.00 & 1.50 & - & - \\ .25 & .15 & .25 & 1.25 \\ .10 & - & - & .50 \\ .25 & .15 & .25 & 1.25 \\ .15 & .10 & .15 & .75 \\ .20 & .10 & .20 & 1.00 \\ .15 & .10 & - & .75 \\ .15 & -10 & - & -75 \\ .15 & .10 & - & .50 \\ .10 & .05 & - & .75 \\ .15 & .10 & - & .75 \\ .15 & .10 & - & \\ .25 & - & - & -\end{array}$

$1.00--$

LONGMEADOW MIXTURE. Contains varieties from the earliest to very latest. There is no trash in this. When I think a variety not worth growing it is destroyed. This comprises many named sorts of recent introduction and some Kunderds "Glory Mixture." You will get full money value.
100 for $\$ 5$
1,000 for $\$ 40$

MEADOWVALE MIXTURE. Contains a fine assortment of Primulinus, named varieties. Make a fine display in the garden. 100 for $\$ 3.50$

25 at 100 rate

1,000 for $\$ 28.00$

250 at 1,000 rate

TERMS: Cash with order unless otherwise arranged. All orders $\$ 1.00$ or over prepaid. EXTRAS given on all cash orders. GUARANTEE: All bulbs are true to name. Mistakes sometimes occur in the rush of handling many orders and various varieties. If you find an error please let me know and I will gladly make it right and more.

Dozen at 10 times single rate.

Hundred at 7 times dozen rate.

Varieties marked * by hundred. 


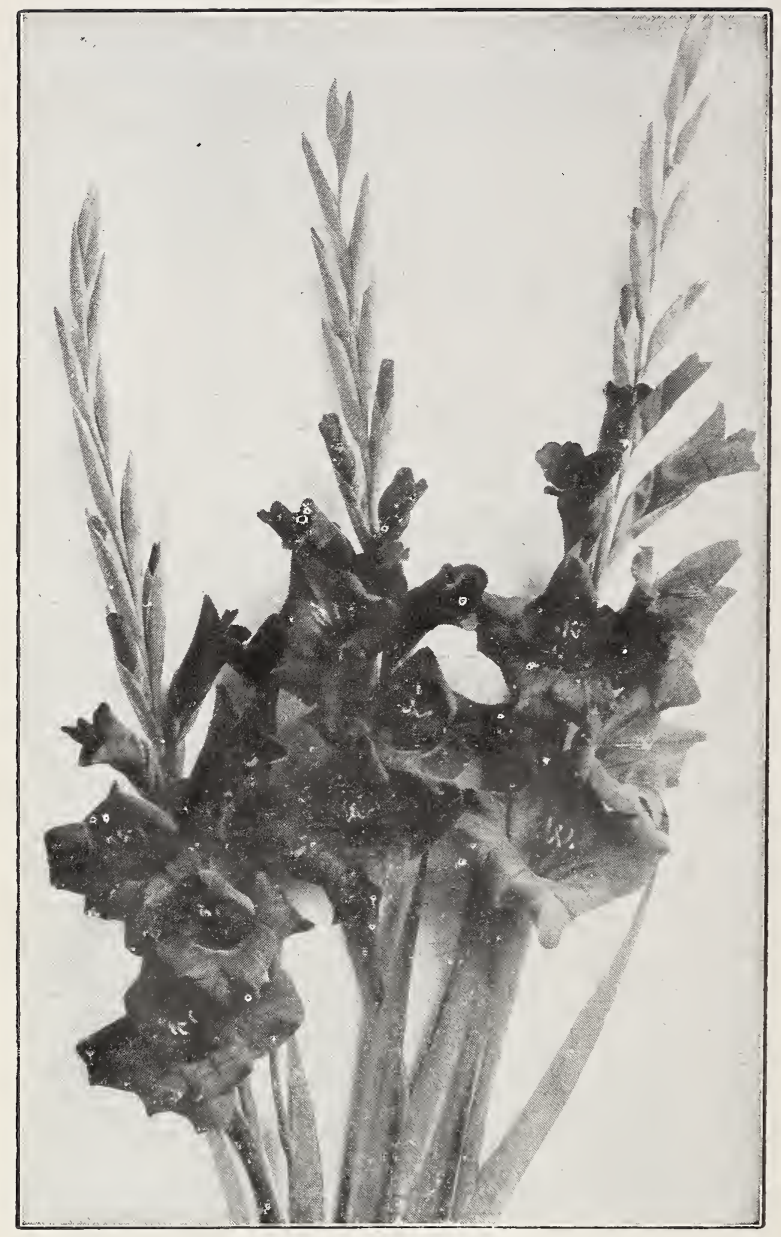

